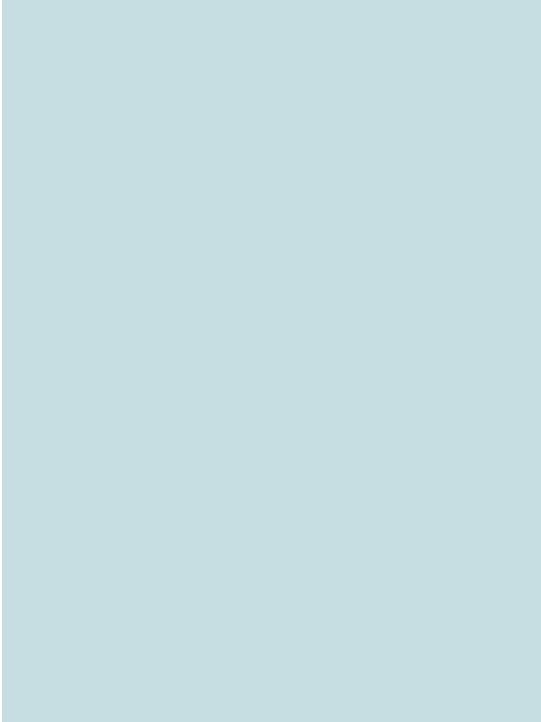

\title{
Development and Validation
} of an Immuno-PET Tracer as a Companion Diagnostic Agent
for Antibody-Drug Conjugate Therapy to Target the CA6 Epitope $^{1}$

Ohad llovich, PhD

Arutselvan Natarajan, PhD

Sharon Hori, PhD

Ataya Sathirachinda, BS

Richard Kimura, PhD

Ananth Srinivasan, PhD

Mathias Gebauer, PhD

Jochen Kruip, PhD

Ingo Focken, PhD

Christian Lange, PhD

Chantal Carrez, PhD

Ingrid Sassoon, PhD

Veronique Blanc, PhD

Susanta K. Sarkar, PhD

Sanjiv S. Gambhir, MD, PhD
${ }^{1}$ From the Department of Radiology (0.I., A.N., S.H., A. Sathirachinda, R.K., A. Srinivasan, S.S.G.) and Departments of Bioengineering and Materials Science \& Engineering (S.S.G.), Stanford University, 318 Campus Dr, Room E153, Stanford, CA 94305; Sanofi R\&D, Biolnnovation Novel Protein Therapeutics, Sanofi-Aventis Deutschland GmbH, Frankfurt, Germany (M.G., J.K., I.F., C.L.); Sanofi Oncology, Vitry, France (C.C., I.S., V.B.); and Sanofi Oncology, Cambridge, Mass (S.K.S.). Received January 21, 2014; revision requested March 12; revision received October 8; accepted November 12; final version accepted December 16 . Supported by Sanofi-Aventis. Address correspondence to S.S.G. (e-mail: sgambhir@stanford.edu).

A.N. and S.H. contributed equally to this work.
Purpose:

Materials and Methods:

Results:

Conclusion:
To develop and compare three copper $64\left({ }^{64} \mathrm{Cu}\right)$-labeled antibody fragments derived from a CA6-targeting antibody (huDS6) as immuno-positron emission tomography (immuno-PET)-based companion diagnostic agents for an antibody-drug conjugate by using huDS6.

Three antibody fragments derived from huDS6 were produced, purified, conjugated to 1,4,7,10-tetraazacyclododecane-1,4,7,10-tetraacetic acid (DOTA), and evaluated in the following ways: (a) the affinity of the fragments and the DOTA conjugates was measured via flow cytometry, (b) the stability of the labeled fragments was determined ex vivo in human serum over 24 hours, and (c) comparison of the in vivo imaging potential of the fragments was evaluated in mice bearing subcutaneous CA6-positive and CA6-negative xenografts by using serial PET imaging and biodistribution. Isotype controls with antilysozyme and anti-DM4 B-Fabs and blocking experiments with an excess of either B-Fab or huDS6 were used to determine the extent of the antibody fragment ${ }^{64} \mathrm{Cu}$-DOTA-B-Fab binding specificity. Immunoreactivity and tracer kinetics were evaluated by using cellular uptake and 48-hour imaging experiments, respectively. Statistical analyses were performed by using $t$ tests, one-way analysis of variance, and Wilcoxon and Mann-Whitney tests.

The antibody fragment ${ }^{64} \mathrm{Cu}$-DOTA-B-Fab was more than 95\% stable after 24 hours in human serum, had an immunoreactivity of more than $70 \%$, and allowed differentiation between CA6-positive and CA6-negative tumors in vivo as early as 6 hours after injection, with a 1.7 -fold uptake ratio between tumors. Isotype and blocking studies experiments showed tracer-specific uptake in antigen-positive tumors, despite some nonspecific uptake in both tumor models.

Three antibody fragments were produced and examined as potential companion diagnostic agents. ${ }^{64} \mathrm{Cu}$-DOTA-BFab is a stable and effective immuno-PET tracer for CA6 imaging in vivo.

${ }^{\circ}$ RSNA, 2015

Online supplemental material is available for this article. 
W ith an antibody-drug conjugate, an antibody is used to deliver a cytotoxic drug selectively by targeting tumor-associated antigens. Two factors have motivated the development of antibody-drug conjugates for cancer therapy. First is the fact that although unmodified antibodies are active, they are rarely curative. The second is the narrow therapeutic window and high prevalence of dose-limiting side effects of some of the most potent nontargeted chemotherapies (1). The transition of antibody-drug conjugates into the clinic would benefit greatly from noninvasive companion diagnostic agents that could potentially allow for patient stratification and early evaluation of effectiveness. Patient stratification according to the expression of membrane-bound antigens typically requires invasive biopsy via fine-needle aspiration of multiple tissue regions to overcome tumor heterogeneity. In addition, decisions regarding patient management are often made by using archived biopsy results that do not necessarily reflect antigen expression at the time of treatment.

SAR566658 (huDS6-DM4) is an antibody-drug immunoconjugate that consists of a humanized monoclonal antibody (huDS6) against the tumorassociated mucin 1-sialoglycotope, CA6 (2), conjugated to the cytotoxic maytansinoid derivative, DM4 (3). CA6 has limited distribution in normal adult tissues but is often overexpressed in carcinomas of the pancreas, ovary, breast, and bladder (4). Upon antibody and/or antigen binding and internalization, the immunoconjugate releases DM4, which binds

\section{Advance in Knowledge}

- The antibody fragment ${ }^{64} \mathrm{Cu}$-DOTA-B-Fab was more than $95 \%$ stable for 24 hours in human serum, was more than $70 \%$ immunoreactive, and illustrated a 1.7-fold higher uptake in CA6-positive versus CA6-negative tumors as early as 6 hours after injection. to microtubules and disrupts the as sembly and/or disassembly dynamics, resulting in mitotic arrest of CA6expressing tumor cells. SAR566658 is currently undergoing phase I clinical trials in patients who have received a diagnosis of CA6-positive ovarian, pancreatic, and breast tumors.

Zirconium 89 ( $\left.{ }^{89} \mathrm{Zr}\right)$-labeled huDS6 has been used successfully for imaging studies of tumors that express CA6 in mice (5). The main disadvantage of using full antibodies for imaging is the prolonged circulation time. Indeed, distinguishing CA6-positive from CA6-negative tumors was possible only 3-6 days after injection of ${ }^{89} \mathrm{Zr}$ huDS6 (5). This requires patients to visit the nuclear medicine department days (or even weeks) apart for tracer administration and imaging. Antibody fragments have shorter biological half-lives, which enables labeling with shorter-lived isotopes (eg, copper 64 $\left[{ }^{64} \mathrm{Cu}\right], t_{1 / 2}=12.7$ hours; and fluorine $18, t_{1 / 2}=110$ minutes) and leads to improved tumor-to-background ratios at earlier time points. The main drawback of using these smaller fragments is that while they usually retain the low nanomolar affinity range of their parent antibodies, their rapid clearance from the blood brings about reduced tumor uptake coupled with increased uptake in the liver and/or kidneys. The purpose of this study was to develop and compare three ${ }^{64} \mathrm{Cu}$-labeled antibody fragments derived from a CA6-targeting antibody (huDS6) as immuno-positron emission tomography (immuno-PET)-based companion diagnostic agents for an antibody-drug conjugate based on huDS6.

\section{Materials and Methods}

Both financial and material (huDS6, B-Fab, cross-over dual variable

\section{Implication for Patient Gare}

- Antibody fragments such as ${ }^{64} \mathrm{Cu}$-DOTA-B-Fab may facilitate same-day imaging by using immuno-PET-based companion diagnostic agents.
[CODV]-Fab, and diabody) support for this publication was provided by Sanofi. O.I. and S.S.G. had control of all the data and information presented in this publication, including any that may present a conflict of interest for the authors who are employees of Sanofi (M.G., J.K., I.F., C.L., C.C., I.S., V.B., and S.K.S.). Research support for O.I. was partially funded by Sanofi.

\section{Cell Lines and Cell Culture}

The human amnion cell line WISH (CA6 positive) was purchased from American Type Culture Collection, Manassas, Va, and the human ovarian carcinoma cell line A2780 (CA6 negative) was purchased from European Cell Culture Collection, Wiltshire, United Kingdom. WISH and A2780 cell lines were cultivated at $37^{\circ} \mathrm{C}$ in humidified $5 \% \mathrm{CO}_{2}$ in Eagle's minimum essential medium or Roswell Park Memorial Institute 1640 medium, respectively, both supplemented with $10 \%$ heat-inactivated fetal bovine serum, $2 \mathrm{mM}(2 \mathrm{mmol} / \mathrm{L})$ L-glutamine, $100 \mathrm{U} / \mathrm{mL}$ penicillin, and $100 \mu \mathrm{g} / \mathrm{mL}$ streptomycin (all from Invitrogen Life

\section{Published online before print \\ 10.1148/radiol.15140058 Content codes: NM MI \\ Radiology 2015; 276:191-198 \\ Abbreviations: \\ CODV $=$ cross-over dual variable \\ DOTA $=1,4,7,10$-tetraazacyclododecane-1,4,7,10 tetraacetic acid \\ HPLC = high-performance liquid chromatography $\mathrm{SEC}=$ size exclusion chromatography}

\section{Author contributions:}

Guarantors of integrity of entire study, 0.I., S.K.S., S.S.G.; study concepts/study design or data acquisition or data analysis/interpretation, all authors; manuscript drafting or manuscript revision for important intellectual content, all authors; approval of final version of submitted manuscript all authors; literature research, 0.I., A.N., A. Srinivasan, V.B., S.K.S., S.S.G.; experimental studies, O.I., A.N., S.H., A. Sathirachinda, R.K., A. Srinivasan, M.G., J.K., I.F., C.L., C.C., I.S., V.B., S.S.G.; statistical analysis, O.I., S.H., S.S.G.; and manuscript editing, O.I., A.N., S.H., R.K., A. Srinivasan, M.G., J.K., I.F., C.L., C.C., I.S., V.B., S.K.S., S.S.G.

\section{Funding:}

This research was supported by the National Institutes of Health (grant P50CA114747).

Conflicts of interest are listed at the end of this article. 


\section{Figure 1}

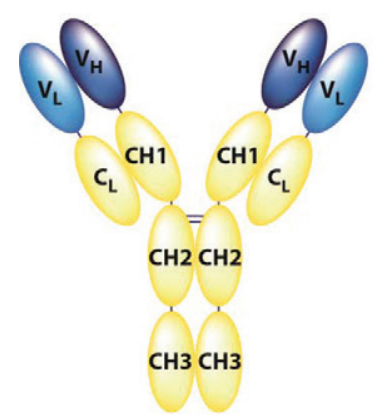

huDS6 - parent antibody

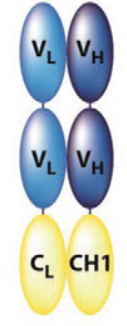

B-Fab

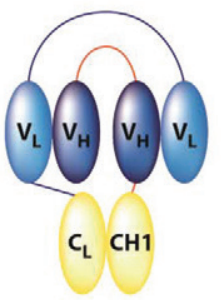

CODV-Fab

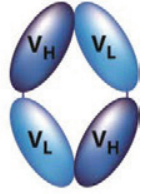

Diabody

Figure 1: Schematic shows the structural relationship between the parent antibody (huDS6) and the engineered fragments B-Fab, CODV-Fab, and diabody. $V_{H}=$ heavy variable domain, $V_{L}=$ light variable domain.

Technologies, Carlsbad, Calif, except for Eagle's minimum essential medium, which was supplied by Lonza, Walkersville, Md).

\section{Construction and Production of Antibody Fragments}

The two Fab fragments (B-Fab and CODV-Fab) were produced by using light variable domain, or $\mathrm{V}_{\mathrm{L}}$, and heavy variable domain, or $\mathrm{V}_{\mathrm{H}}$, sequences from huDS6 (Fig 1). The huDS6 $\mathrm{V}_{\mathrm{L}}$ linker- $\mathrm{V}_{\mathrm{L}}$ and huDS6 $\mathrm{V}_{\mathrm{H}}$-linker- $\mathrm{V}_{\mathrm{H}}$ constructs were then fused at the $3^{\prime}$ end to human immunoglobulin kappa and heavy chain 1 constant domain sequences, respectively. For expression of the huDS6 diabody, the respective $\mathrm{V}_{\mathrm{L}}$ and $\mathrm{V}_{\mathrm{H}}$ sequences from huDS6 were arranged in tandem and separated by a linker sequence (Fig 1). To allow radiolabeling via $\mathrm{C}$-terminal cysteine and purification with nickel-chelate chromatography, the DS6 diabody construct was fused at the $3^{\prime}$ end to sequence GGCGGHHHHHH. B-Fab isotype controls, which have a similar structure to DS6-B-Fab but target hen egg white lysozyme or the DM4 drug moiety instead of CA6, were used to evaluate binding specificity. Isotype controls were expressed by using the $\mathrm{V}_{\mathrm{L}}$ and $\mathrm{V}_{\mathrm{H}}$ sequences of antibody D1.3 directed against hen egg white lysozyme (6) or an internally generated anti-DM4 antibody in the same manner as the DS6-B-Fab. All antibody fragment sequences were cloned into the episomal expression vector $\mathrm{pXL}$, an analog of the pTT vector (7).

\section{Flow Cytometry}

WISH or A2780 cells $\left([3\right.$ to 5$\left.] \times 10^{5}\right)$ were resuspended in $0.1 \mathrm{~mL}$ binding buffer (phosphate-buffered saline, 1\% bovine serum albumin) containing 1:3 dilutions of $3 \times 10^{-7}$ to $1 \times 10^{-10} \mathrm{M}$ $\left(3 \times 10^{-7}\right.$ to $\left.1 \times 10^{-10} \mathrm{~mol} / \mathrm{L}\right)$ of the antibody fragment (or its 1,4,7,10-tetraazacyclododecane-1,4,7,10-tetraacetic acid [DOTA] conjugate) and kept for 1 hour on ice. Cells were washed twice with binding buffer and incubated for 1 hour on ice in the dark with either Alexa Fluor 488-conjugated mouse antihuman kappa mAb (1:50, Invitrogen Life Technologies) or fluorescein isothiocyanateconjugated anti-6X His tag antibody (1:100, Abcam, Cambridge, Mass), for Fab fragments and diabody respectively, in $0.1 \mathrm{~mL}$ binding buffer. After three washes, cells were resuspended in 0.2 $\mathrm{mL}$ of phosphate-buffered saline that contained $1 \%$ formaldehyde, and flow cytometry was performed immediately.

Flow cytometry data were analyzed by using GraphPad Prism 6 (GraphPad, San Diego, Calif), and affinity was calculated on the basis of a one-site model of binding.

\section{DOTA Conjugation and Radiolabeling}

DOTA was chosen over other chelators with potentially higher stability because of its widespread use and its approval by the U.S. Food and Drug Administration, facilitating future clinical translation. DOTA conjugation to antibody fragments was performed according to established protocols (8) by using metalfree buffers. The diabody was reduced by using dithiothreitol and reacted with 1,4,7,10-tetraazacyclododecane-1,4,7tris-acetic acid-10-maleimidoethylacetamide (Maleimido-monoamide-DOTA; Macrocyclics, Dallas, Tex) as described previously (9).

The mean DOTA-fragment ratio was determined by using the change in mass seen in Matrix-Assisted Laser Desorption Ionization (AB Sciex 5800 TOF/TOF machine [AB Sciex, Framingham, Mass] equipped with a CovalX high-mass detector, 1 pM [1pmol/L] bovine serum albumin used as an internal standard) divided by the mass of a single DOTA substituent.

The pH-balanced ${ }^{64} \mathrm{CuCl}_{2}$ (approximately $135 \mathrm{MBq}$ in $0.1 \mathrm{M}[0.1 \mathrm{~mol} / \mathrm{L}]$ $\mathrm{HCl}$, University of Wisconsin-Madison, Madison, Wis) and the DOTAconjugated antibody fragment (100 $\mu \mathrm{g})$ were incubated at $37^{\circ} \mathrm{C}$ in ammonium acetate $(200-300 \mu \mathrm{L}, 0.1 \mathrm{M}[0.1$ $\mathrm{mol} / \mathrm{L}]$, pH level of 5.5) for 1 hour with gentle shaking at 300 revolutions per minute. Ethylenediaminetetraacetic acid $(0.5 \mathrm{M}[0.5 \mathrm{~mol} / \mathrm{L}], \mathrm{pH}$ level of 8) was added to a final concentration of $0.01 \mathrm{M}(0.01 \mathrm{~mol} / \mathrm{L})$, and the incubation continued at room temperature for another 15 minutes. The reaction was purified via size exclusion chromatography (SEC) high-performance liquid chromatography (HPLC) (SECS2000; Phenomenex, Torrance, Calif) to give the purified tracer formulated in phosphate buffer $(0.1 \mathrm{M}[0.1$ $\mathrm{mol} / \mathrm{L}]$, pH level of 6.9).

Radiochemical purity was determined by using both SEC HPLC and instant thin-layer chromatography with Tec-Control Chromatography strips (Biodex Medical Systems, Shirley, NY) developed in saline.

\section{Human Serum Stability}

The ${ }^{64} \mathrm{Cu}$-labeled fragments in phosphate buffer were mixed with a ninefold volume of human serum (Equitech-Bio, Kerrville, Tex) and incubated at $37^{\circ} \mathrm{C}$ for 24 hours. Activity was analyzed via cellulose acetate 
electrophoresis performed with barbital buffer $(0.05 \mathrm{M}[0.5 \mathrm{~mol} / \mathrm{L}], \mathrm{pH}$ level of 8.6) or SEC HPLC (SEC-S3000; Phenomenex) with 1-minute fractions counted in a gamma ray counter.

\section{Tumor Xenograft Murine Model}

Animal experiments were conducted in accordance with federal and institutional regulations by using a protocol approved by the Stanford University Institutional Animal Care and Use Committee. Three million WISH or A2780 cells in a mixture of phosphate-buffered saline and growth factor-reduced matrigel (ratio of $1: 1,100 \mu \mathrm{L}$ ) were subcutaneously injected into the shoulders of 6-8-week-old female nu/nu mice. Xenografted tumors were grown for 12-13 days (until they reached a volume of 150-350 $\mathrm{mm}^{3}$ ) (10). Four or five animals were used in both WISH and A2780 groups in all the imaging and biodistribution studies except for blocking studies, where three to five animals were used.

To determine CA6 expression, excised tumors (WISH, $n=5$; A2780, $n=3$ ) were placed in formalin, and radioactivity was allowed to decay. Tumors were paraffin embedded, and $5-\mu \mathrm{m}$ sections were stained for CA6 expression by using huDS6 as the primary antibody. Staining clearly showed high expression of CA6 in WISH tumors but not in A2780 tumors (Fig E1 [online]).

\section{Cell Binding and Immunoreactive Fraction Analysis}

The immunoreactive fraction was calculated as described previously (11) with a 4:1 silicone oil-mineral oil mixture in place of the described phthalate oil mixture. WISH cells $\left(3 \times 10^{5}\right.$ to $6 \times 10^{6}$ cells) were used to determine immunoreactivity. A2780 cells $\left(3 \times 10^{6}\right.$ cells $)$ were used to validate the specificity of the binding of the tracer to CA6-positive cells.

\section{Small-Animal PET Imaging and Biodistribution}

Mice were anesthetized with $2 \%$ isoflurane in oxygen at $2 \mathrm{~L} / \mathrm{min}$, and 1.8-3.2 $\mathrm{MBq}$ of tracer was injected intravenously via the tail vein. Five-minute static PET scans were performed at $1,4,6,12$, and 24 hours after injection by using a micro-PET/computed tomographic scanner (1.4-mm resolution, Inveon; Siemens, Malvern, Pa), followed by biodistribution. In the 48-hour imaging study, $5.5-7.4 \mathrm{MBq}$ of ${ }^{64} \mathrm{Cu}^{-}$ DOTA-B-Fab was injected, and imaging was performed $4,8,17,28,41$, and 47 hours after injection.

Live-animal blocking studies and two isotype controls (each having a structure similar to that of DS6-B-Fab but targeting antigens not found in our animal model) were used to show the specificity of the uptake of the B-Fab fragment for WISH tumors. Blocking was achieved with intravenous administration of unmodified B-Fab or huDS6 prior to that of the tracer. B-Fab (2 mg, $180 \mu \mathrm{L}$ ) was injected either 2.5 or 24 hours prior to the tracer, while huDS6 (1 mg, $100 \mu \mathrm{L}$ ) was injected either 4 or 25 hours prior to the tracer.

\section{Micro-PET Image Data Analysis}

Regions of interest were drawn by the lead author (O.I., with 3 years of experience) by using the image analysis software IRW (Siemens). Regions of interest were drawn for tumors (150$\left.350 \mathrm{~mm}^{3}\right)$, liver $\left(200-400 \mathrm{~mm}^{3}\right)$, and kidneys (130-190 $\mathrm{mm}^{3}$ ) on the basis of CT data and centered on the peak-ofactivity profile. Background regions of interest $\left(45-100 \mathrm{~mm}^{3}\right)$ were drawn on the contralateral shoulder. Regions of interest for tumors and kidneys were maximized to the size of the organ. Radioactivity distribution in the liver was homogeneous; therefore, a large representative region of interest was deemed sufficient for analytical purposes. Data (percentage injected dose per gram of tissue $[\mathrm{ID} / \mathrm{g}]$ ) were corrected for partial volume effect and spillover (12) by using calibration factors obtained from scanning a cylinder that contained phantoms of different sizes, with phantom-to-background activity ratios ranging from $3: 1$ to $25: 1$ (13). Image quality was compared at different time points after injection to determine whether imaging at early time points was feasible.

\section{Statistical Analysis}

Two-sample comparisons (of B-Fab, CODV-Fab, and diabody biodistributions) were determined by using a two-tailed $t$ test for unequal variances. A one-way analysis of variance test and a post hoc Dunnett multiple-comparisons test was used for the blocking experiment, with the unblocked WISH group used as control. Isotype control experiments were tested with exact Wilcoxon and Mann-Whitney tests. A Bonferroni-adjusted significance level of .0083 was used.

Analyses were performed in Prism 6 (GraphPad, version 6.02; GraphPad). Data points were presented as means \pm standard deviations. Statistical significance was denoted for a level of $P$ $<.05$ in any single-comparison statistical tests.

\section{Results}

\section{Production and Characterization of Antibody Fragments}

$\mathrm{B}-\mathrm{Fab}$ and CODV-Fab were purified from HEK293 cultures with a mean yield of approximately 3 and $2 \mathrm{mg} / \mathrm{L}$ supernatant, respectively, whereas the yield of the diabody was lower $(1 \mathrm{mg} / \mathrm{L})$. Analysis of the purified proteins with sodium dodecyl sulfate polyacrylamide gel electrophoresis demonstrated that the two-step purification yielded Fab fragments with more than $95 \%$ purity and diabody with approximately $90 \%$ purity (Fig E2 [online]).

Homogeneity of all antibody fragments was checked with analytical gel filtration, and identity was confirmed with mass spectrometry (Fig E3 [online]).

\section{Binding Analysis of Antibody Fragments with DOTA Conjugates}

All fragments exhibited low nanomolar $K_{d}$ values $(4-13 \mathrm{nM}$ [4-13 nmol/L]) on WISH cells, which were comparable to those of the parent antibody huDS6 $\left(K_{d}=2\right.$ $\mathrm{nM}[2 \mathrm{nmol} / \mathrm{L}])$ but showed no detectable binding to A2780 cells (Table 1). The affinities of the DOTA conjugates were similar to those of the corresponding parent fragments, ensuring that 


\section{Table 1}

\begin{tabular}{|c|c|c|c|c|}
\hline Fragment & $\begin{array}{l}K_{d} \text { on WISH } \\
\text { Cells }(\mathrm{nM})^{*}\end{array}$ & $\begin{array}{l}\text { DOTA-Fragment } \\
\text { Ratio }\end{array}$ & $\begin{array}{l}\text { Purity (SEC } \\
\text { HPLC) (\%) }\end{array}$ & $\begin{array}{l}\text { Purity (SDS } \\
\text { PAGE) (\%) }\end{array}$ \\
\hline huDS6 & $2.13 \pm 0.4$ & $\ldots$ & $>99$ & $>95$ \\
\hline B-Fab & $4 \pm 1.4$ & $\ldots$ & $>95$ & $>95$ \\
\hline DOTA-B-Fab & $7.36 \pm 1.1$ & 2.35 & $>97$ & $>95$ \\
\hline${ }^{64} \mathrm{Cu}-\mathrm{DOTA}$-B-Fab & $16.3 \pm 6.9$ & 2.35 & $>97$ & $>95$ \\
\hline CODV-Fab & $13.2 \pm 9.8$ & $\ldots$ & $>95$ & $>95$ \\
\hline DOTA-CODV-Fab & $20 \pm 3$ & 2 & $>99$ & $>95$ \\
\hline Diabody & $9.73 \pm 1.8$ & $\ldots$ & $>95$ & $>95$ \\
\hline DOTA-diabody & $7.52 \pm 2.8$ & 1.52 & $\sim 95$ & $>90$ \\
\hline Lysozyme-B-Fab & ND & $\ldots$ & $>95$ & $>90$ \\
\hline DOTA-lysozyme-B-Fab & ND & 2.57 & $>98$ & $>90$ \\
\hline DM4-B-Fab & ND & $\ldots$ & $>95$ & $>95$ \\
\hline DOTA-DM4-B-Fab & ND & 2.29 & $>99$ & $>95$ \\
\hline
\end{tabular}

Note.-To convert nanomolar to nanomoles per liter, multiply by 1. ND = not detected, PAGE = polyacrylamide gel electrophoresis, SDS $=$ sodium dodecyl sulfate.

* Data are mean \pm standard error of three replicates.

DOTA derivatization did not adversely affect binding affinity. ${ }^{64} \mathrm{Cu}$-DOTA-BFab was stored at $4^{\circ} \mathrm{C}$ for a week to allow for radioactivity decay and was found to also retain its affinity to a large degree (Fig 2). As expected, the isotype control B-Fab fragments lacked binding to both cell types.

\section{DOTA Conjugation, Radiolabeling, and Human Serum Stability}

The Fab fragments were successfully and reproducibly conjugated to DOTA to attain between 1.5 and 2.5 DOTA substitutions per antibody fragment (Table 1). The radiolabeling efficiency of all Fab fragments was $50 \%-80 \%$, and the radiochemical purity was more than 97\%. Specific activities ranged from 55 to $75 \mathrm{GBq} / \mu \mathrm{mol}$. The radiolabeling efficiency for the diabody was only $10 \%-$ $15 \%$, and the specific activity was approximately $14 \mathrm{GBq} / \mu \mathrm{mol}$, which was as good as previously published work in which ${ }^{64} \mathrm{Cu}$-labeled diabodies were used $(14,15)$.

The labeled diabody was found to be highly unstable after 24 hours in human serum, with less than $50 \%$ of the radioactivity associated with its monomeric form. The CODV-Fab showed mixed results, with stabilities ranging from $55 \%$ to $90 \%$. All B-Fabs (DS6, lysozyme, and DM4) had more than 95\% of the activity in monomeric form (Fig E4 [online]).

After selecting the B-Fab for further evaluation, DOTA conjugation and labeling of $\mathrm{B}-\mathrm{Fab}$ were optimized to enable the synthesis of high-specificactivity $(>75 \mathrm{GBq} / \mu \mathrm{mol}){ }^{64} \mathrm{Cu}$-DOTA$\mathrm{B}-\mathrm{Fab}$ by using only $0.9-1.2$ DOTAs per B-Fab.

\section{Cell Binding and Immunoreactive Fraction Analysis}

WISH cells bound $48 \% \pm 1.6$ of the ${ }^{64} \mathrm{Cu}-\mathrm{DOTA}-\mathrm{B}-\mathrm{Fab}$ at the highest concentration of cells tested. Addition of BFab prior to ${ }^{64} \mathrm{Cu}$-DOTA-B-Fab blocked more than $90 \%$ of binding at all cellular concentrations used. A2780 cells bound less than $1 \%$ of the tracer, regardless of prior treatment with B-Fab. The immunoreactive fraction of ${ }^{64} \mathrm{Cu}$-DOTA-B-Fab was calculated to be more than $70 \%$ ( $n$ = 2) (Fig E5 [online]).

\section{Biodistribution and Imaging in Tumor-bearing Mice}

Table 2 shows the biodistribution results for the organs of highest uptake at 24 hours, while the full biodistribution data are available in Table E1

\section{Figure 2}

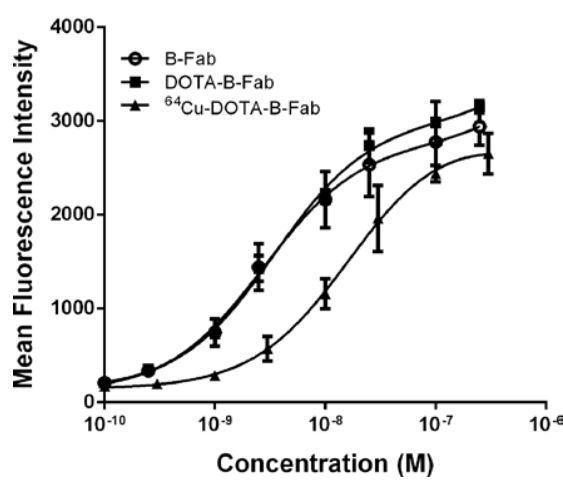

Figure 2: Binding curves for B-Fab, DOTA-B-Fab, and ${ }^{64} \mathrm{Cu}-\mathrm{DOTA}-\mathrm{B}-\mathrm{Fab}$ show that DOTA conjugation and ${ }^{64} \mathrm{Cu}$ labeling caused a slightly decreased affinity to CA6-positive cells. Mean fluorescence intensity is plotted versus the concentration of antibody fragment used for staining. $M=$ molar.

(online). Six hours after the injection of tracer, tumors could be visualized for all fragments with clear delineation of CA6-positive and CA6-negative tumors and little residual blood activity (Fig 3 ). The tumor, liver, kidneys, and bladder were the only prominent organs visible above background at 24 hours (Figs E6 and E7 [online] and Movies 1 and 2 [online]), but the overall quality of the image was not superior to that obtained at 6 hours. Biodistribution data showed that the diabody and Fab fragments had their highest uptake values in the kidneys (112 and 50\%-60\% ID/g, respectively), followed by liver uptake (10\%-15\% ID/g) and CA6-positive tumors (4\%-8\% ID/g) (Table 2). The CODV-Fab and B-Fab had a larger difference (1.42-fold, $P=.0014$; and 1.7fold, $P=.0021$, respectively) between CA6-positive and CA6-negative tumors compared with the diabody (1.26-fold, $P<$.033). Imaging beyond the 28hour time point proved unnecessary, owing to decreasing tumor-to-background ratio without improvement of the WISH-A2780 uptake ratio (Fig E8 [online]).

In all but one blocking study, it was possible to significantly reduce tracer uptake by 24\%-26\% (analysis 


\section{Table 2}

\section{Biodistribution Studies Performed 24 Hours after Injection of Radiolabeled Fragments in Mice Bearing Subcutaneous WISH or A2780} Tumors

\begin{tabular}{lllllc} 
Fragment & $\begin{array}{l}\text { WISH Tumor } \\
\text { (percentage ID/g) }\end{array}$ & $\begin{array}{l}\text { A2780 Tumor } \\
\text { (percentage ID/g) }\end{array}$ & $\begin{array}{l}\text { WISH-A2780 } \\
\text { Ratio }\end{array}$ & $\begin{array}{l}\text { Liver } \\
\text { (percentage ID/g) }\end{array}$ & $\begin{array}{l}\text { Kidneys } \\
\text { (percentage ID/g) }\end{array}$ \\
\hline${ }^{64} \mathrm{Cu}-$ DOTA-B-Fab & $7.17 \pm 1.6$ & $4.6 \pm 0.48$ & 1.56 & $10.7 \pm 1.79$ & $66 \pm 2$ \\
${ }^{64} \mathrm{Cu}-$ DOTA-CODV-Fab & $6.85 \pm 0.42$ & $4.84 \pm 0.69$ & 1.42 & $14.6 \pm 1.8$ & $54 \pm 6.4$ \\
${ }^{64} \mathrm{Cu}-$ DOTA-diabody & $4.35 \pm 0.53$ & $3.25 \pm 0.2$ & 1.34 & $9.55 \pm 0.34$ & $112 \pm 19$ \\
${ }^{64} \mathrm{Cu}-$ DOTA-antilysozyme-B-Fab & $4.84 \pm 1.02$ & $3.84 \pm 0.49$ & 1.26 & $15.2 \pm 1.3$ & $70 \pm 9.6$ \\
${ }^{64} \mathrm{Cu}-$ DOTA-anti-DM4-B-Fab & $4.04 \pm 0.43$ & $3.03 \pm 0.24$ & 1.33 & $10.4 \pm 1.6$ & $52 \pm 8.3$ \\
\hline
\end{tabular}

Note.-Percentage ID/g data are presented as means \pm standard deviations.

${ }^{*} n=4-5$ for all biodistribution experiments.

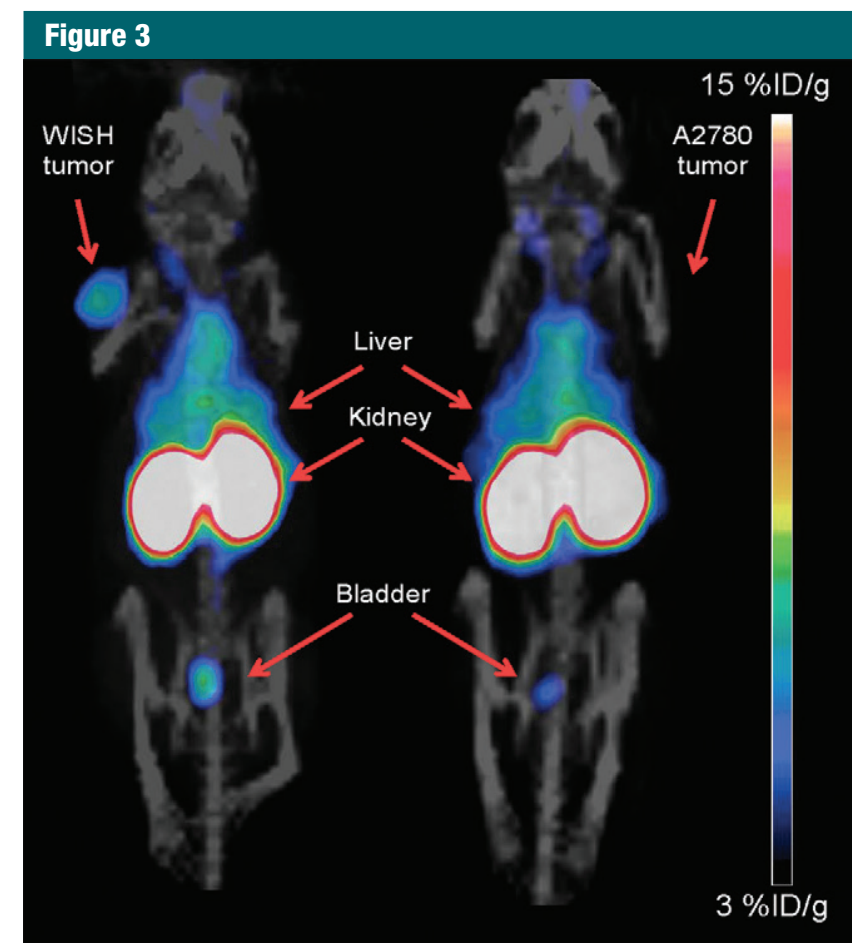

Figure 3: Maximum-intensity projections of coronal whole-body PET/CT images were acquired 6 hours after injection of ${ }^{64} \mathrm{Cu}$-DOTA-B-Fab in mice bearing either WISH (left) or A2780 (right) subcutaneous tumors. The tumors, liver, kidneys, and bladder are labeled. Scale bar represents a range of $3 \%-15 \% \mathrm{ID} / \mathrm{g}$.

of variance, $F[4,14]=4.692[P<.05]$; Dunnett test, $P=.91$ for B-Fab at 24 hours, $P=.025$ for B-Fab at 2 hours, $P=.016$ for DS6 at 4 hours, and $P$ $=.031$ for DS6 at 24 hours; Fig 4). Uptake of both isotype controls into WISH tumors was significantly lower than the original B-Fab $(P<$ .001 , Table 2); however, uptake of the two isotype controls did not differ significantly from one another $(P=.286)$.

\section{Discussion}

Companion diagnostic agents could enable patient stratification on the basis of antigen expression, aiding physicians in identifying patients who might best respond to targeted therapies. Such tools may increase the effect and relevance of data generated from clinical studies and help streamline drug development according to U.S. Food and Drug Administration requirements for targeted therapies $(16,17)$. Nuclear medicine-based companion diagnostic agents, particularly for membranebound antigens, have been increasing in popularity in recent years. Two examples of success in the field are the folate-receptor-targeting single photon emission computed tomography tracer technetium 99m EC20, used to predict response to therapy with antifolates (18), and the human epidermal growth factor receptor 2-targeting PET tracer ${ }^{89} \mathrm{Zr}$-trastuzumab, used to detect human epidermal growth factor receptor 2-positive breast cancer lesions (19).

When considering the in vivo behavior of the three fragments, the fast blood clearance of the diabody makes it a good candidate for same-day imaging in patients. However, its low stability in human serum would likely make clinical translation difficult. Conversely, the BFab fragment exhibited consistent stability in human serum, good expression in HEK293 cells, a high WISH-A2780 uptake ratio, and the ability to image mice as early as 6 hours after injection, thus emerging as our lead choice for optimization and further evaluation.

${ }^{64} \mathrm{Cu}$-DOTA-B-Fab showed the highest and most consistent serum stability. It also showed high immunoreactivity 


\section{Figure 4}

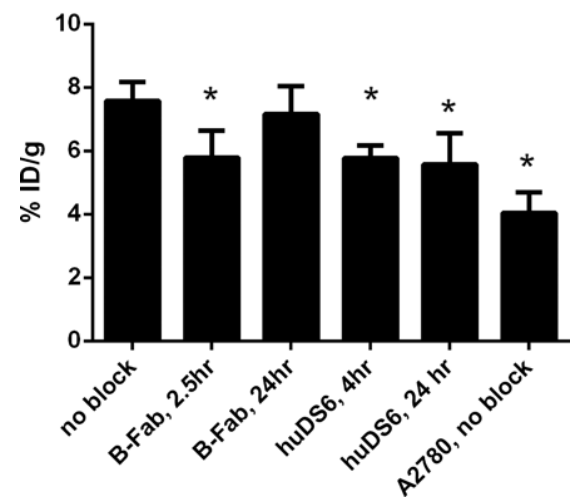

Figure 4: Graph demonstrates the effect of different blocking approaches on ${ }^{64} \mathrm{Cu}$-DOTA-B-Fab uptake in mice bearing WISH subcutaneous tumors. $*=P<.05$. All blocking protocols but one were successful in decreasing WISH tumor uptake in a statistically significant manner. Error bars represent the standard deviation of 3-4 replicates.

$(>70 \%)$ and specific uptake in WISH cells, which could be blocked efficiently $(>90 \%)$ by means of preincubation with the unlabeled B-Fab. Binding to A2780 cells was negligible $(<1 \%)$.

Twenty-four hours after tracer injection, both Fab fragments achieved tumor-to-blood ratios of more than 4 and tumor-to-muscle ratios of more than 8 , compared with ratios of 2 and 4 , respectively, for the diabody. Among the three derivatives, ${ }^{64} \mathrm{Cu}$-DOTA-B-Fab provided the highest WISH tumor uptake and highest WISH-A2780 uptake ratio. Obviously, a portion of the signal from both WISH and A2780 tumors is due to free and nonspecifically bound ${ }^{64} \mathrm{Cu}$-DOTA-B-Fab. The high renal uptake and retention of ${ }^{64} \mathrm{Cu}$-DOTA-B-Fab in the mouse model is likely due to the catabolism of the tracer in kidney cells and retention of the ${ }^{64} \mathrm{Cu}$-labeled metabolites. On the basis of these results, ${ }^{64} \mathrm{Cu}$-DOTA-B-Fab was chosen for further evaluation and was assessed for binding specificity in both cellular uptake and live animal studies.

Blocking was performed with both B-Fab and huDS6 by using large fold excesses due to experience with ${ }^{89} \mathrm{Zr}$ huDS6, which showed minimal blocking (approximately 15\% reduction in tumor uptake with a tenfold excess).
B-Fab administered 24 hours prior to the tracer failed to block tracer uptake; this may be due to its short biological half-life and high CA6 recycling rate. Isotype controls showed that despite having some nonspecific binding, the binding of ${ }^{64} \mathrm{Cu}$-DOTA-B-Fab to WISH cells was indeed specific, as the uptake of DS6-derived B-Fab was significantly higher than the anti-DM4 and antilysozyme B-Fabs $(P<.0001$ and $P<.001$, respectively), while the latter two did not differ significantly from each other $(P=.29)$.

Two main concerns for using ${ }^{64} \mathrm{Cu}$ DOTA-B-Fab are its relatively low ratio between CA6-positive and CA6-negative tumors and its proportion of nonspecific binding. Regarding the low ratio, immunohistochemistry performed on WISH tumors showed that CA6 expression in vivo decreased over time (Fig E9 [online]). Less than $55 \%$ of the nonnecrotic WISH tumor tissue was positive for CA6 expression 10 days after tumor implantation. The limited CA6 expression may produce less impressive preclinical results but should be more reflective of the challenges that clinical translation will present.

Regarding the nonspecific binding of ${ }^{64} \mathrm{Cu}$-DOTA-B-Fab, fast-growing tumors require very quick growth of angiogenic vessels. Fast-growing angiogenesis displays leaky fenestrations that can lead to increased enhanced permeability and retention effect and more nonspecific uptake of large $(>40-\mathrm{kDa})$ particles. Goutayer et al have shown that although fast-growing tumors have higher accumulation of large particles relative to slow-growing tumors, this is mainly due to nonspecific enhanced permeability and retention effect and also results in partial loss of accumulation specificity (20).

In light of these concerns, one must keep in mind that ${ }^{89} \mathrm{Zr}$-huDS6 produced a CA6-positive to CA6-negative tumor uptake ratio of 3 , moderate uptake in A2780 tumors (approximately $8 \% \mathrm{ID} / \mathrm{g}$ ), and low blocking effectiveness (reduction of approximately 13\% of WISH tumor uptake) 3 days after injection of the tracer in the same tumor models. A full antibody and its fragments sharing similar limitations may reinforce our doubts of the current tumor model, and future preclinical studies will involve the use of slowgrowing, stable, CA6-expressing tumor models to address these concerns.

Overcoming all these obstacles, ${ }^{64} \mathrm{Cu}$-DOTA-B-Fab showed high binding affinity, stability, and a promising ability to allow differentiation between CA6-positive and CA6-negative tumors in vivo at early time points after injection, suggesting that it may be a successful companion diagnostic agent for antibody-drug conjugate therapy.

Acknowledgments: We thank the Stanford Small Animal Imaging Facility and especially Frezghi Habte, PhD, for his help with image analysis.

Disclosures of Conflicts of Interest: O.I. Ac tivities related to the present article: author received a grant and nonfinancial support from Sanofi. Activities not related to the present ar ticle: disclosed no relevant relationships. Other relationships: author has a patent. A.N. Activities related to the present article: disclosed no relevant relationships. Activities not related to the present article: disclosed no relevant relationships. Other relationships: author has patents pending. S.H. disclosed no relevant relationships. A. Sathirachinda Activities related to the present article: author received a grant and nonfinancial support from Sanofi. Activities not related to the present article: disclosed no relevant relationships. Other relationships: dis closed no relevant relationships. R.K. Activities related to the present article: author received payment from Sanofi Aventis. Activities not re lated to the present article: disclosed no relevant relationships. Other relationships: author has a patent pending. A. Srinivasan disclosed no relevant relationships. M.G. Activities related to the present article: disclosed no relevant relationships. Activities not related to the present article: author is an employee of Sanofi; author has received payment for patents and has stock and/ or stock options in Sanofi. Other relationships: author has a patent. J.K. Activities related to the present article: disclosed no relevant relationships. Activities not related to the present article: author is an employee of Sanofi; author received payment from Sanofi for a patent and has stock and/or stock options in Sanofi. Other relationships: disclosed no relevant relationships. I.F. Activities related to the present article: disclosed no relevant relationships. Activities not related to the present article: author is an employee of Sanofi and has patents with and stock and/or stock options in Sanofi. Other relationships: disclosed no relevant relationships. C.L. Activities related to the present article: disclosed no relevant relationships. Activities not related to the present article: author is an employee of Sanofi; author received payment from Sanofi for a patent. Other relationships: 
disclosed no relevant relationships. C.C. Activities related to the present article: disclosed no relevant relationships. Activities not related to the present article: author is an employee of Sanofi. Other relationships: disclosed no relevant relationships. I.S. Activities related to the present article: disclosed no relevant relationships. Activities not related to the present article: author is an employee of Sanofi. Other relationships: disclosed no relevant relationships. V.B. Activities related to the present article: disclosed no relevant relationships. Activities not related to the present article: author is an employee of Sanofi. Other relationships: disclosed no relevant relationships. S.K.S. Activities related to the present article: disclosed no relevant relationships. Activities not related to the present article: author is an employee of Sanofi. Other relationships: disclosed no relevant relationships. S.S.G. Activities related to the present article: author received a grant and nonfinancial support from Sanofi. Activities not related to the present article: disclosed no relevant relationships. Other relationships: author has a patent.

\section{References}

1. Blum RH, Wittenberg BK, Canellos GP, et al. A therapeutic trial of maytansine. Cancer Clin Trials 1978;1(2):113-117.

2. Kearse KP, Smith NL, Semer DA, et al. Monoclonal antibody DS6 detects a tumorassociated sialoglycotope expressed on human serous ovarian carcinomas. Int J Cancer 2000;88(6):866-872.

3. Lopus M, Oroudjev E, Wilson L, et al. Maytansine and cellular metabolites of antibodymaytansinoid conjugates strongly suppress microtubule dynamics by binding to microtubules. Mol Cancer Ther 2010;9(10):26892699 .

4. Smith NL, Halliday BE, Finley JL, Wennerberg AE. The spectrum of immunohistochemical reactivity of monoclonal antibody DS6 in nongynecologic neoplasms. Appl Immunohistochem Mol Morphol 2002;10(2):152-158.

5. Jouannot E, Besret B, D’Heilly S, et al Shedding light on antibody drug conjugates: preclinical immunoPET studies using $89 \mathrm{Zr}$ huDS6-DM4 (SAR566658). Kyoto, Japan: WMIC, 2011.

6. Freire E. The propagation of binding interactions to remote sites in proteins: analysis of the binding of the monoclonal antibody D1.3 to lysozyme. Proc Natl Acad Sci U S A 1999;96(18):10118-10122.

7. Durocher Y, Perret S, Kamen A. High-level and high-throughput recombinant protein production by transient transfection of suspension-growing human 293-EBNA1 cells. Nucleic Acids Res 2002;30(2):E9.

8. Cooper MS, Ma MT, Sunassee K, et al. Com parison of (64)Cu-complexing bifunctional chelators for radioimmunoconjugation: labeling efficiency, specific activity, and in vitro/in vivo stability. Bioconjug Chem 2012;23(5): 1029-1039.

9. Sirk SJ, Olafsen T, Barat B, Bauer KB, Wu AM. Site-specific, thiol-mediated conjugation of fluorescent probes to cysteine-modified diabodies targeting CD20 or HER2. Bioconjug Chem 2008;19(12):2527-2534.

10. Tomayko MM, Reynolds CP. Determination of subcutaneous tumor size in athymic (nude) mice. Cancer Chemother Pharmacol 1989; 24(3):148-154

11. Rowlinson-Busza G. Determination of the immunoreactivity of radiolabeled monoclonal antibodies. Methods Mol Med 2000;40: 351-361.

12. Soret M, Bacharach SL, Buvat I. Partialvolume effect in PET tumor imaging. J Nucl Med 2007;48(6):932-945.

13. Sakaguchi Y, Mizoguchi N, Mitsumoto T, et al. A simple table lookup method for
PET/CT partial volume correction using a point-spread function in diagnosing lymph node metastasis. Ann Nucl Med 2010;24 (8) :585-591 .

14. McCabe KE, Liu B, Marks JD, Tomlinson JS, Wu H, Wu AM. An engineered cysteinemodified diabody for imaging activated leukocyte cell adhesion molecule (ALCAM)-positive tumors. Mol Imaging Biol 2012;14(3): 336-347.

15. Olafsen T, Cheung CW, Yazaki PJ, et al. Covalent disulfide-linked anti-CEA diabody allows site-specific conjugation and radiolabeling for tumor targeting applications. Protein Eng Des Sel 2004;17(1):21-27.

16. Hamburg MA, Collins FS. The path to personalized medicine. N Engl J Med 2010;363(4): 301-304.

17. Papadopoulos N, Kinzler KW, Vogelstein $\mathrm{B}$. The role of companion diagnostics in the development and use of mutation-targeted cancer therapies. Nat Biotechnol 2006; 24(8):985-995

18. Symanowski JT, Maurer AH, Naumann RW, Shah NP, Morgenstern D, Messmann RA Use of 99mTc-EC20 (a folate-targeted imaging agent) to predict response to therapy with EC145 (folate-targeted therapy) in advanced ovarian cancer. ASCO Meeting Abstracts; 2010; 5034

19. Dijkers EC, Oude Munnink TH, Kosterink JG, et al. Biodistribution of $89 \mathrm{Zr}$-trastuzumab and PET imaging of HER2-positive lesions in patients with metastatic breast cancer. Clin Pharmacol Ther 2010;87(5):586592.

20. Goutayer M, Dufort S, Josserand V, et al. Tumor targeting of functionalized lipid nanoparticles: assessment by in vivo fluorescence imaging. Eur J Pharm Biopharm 2010;75(2) 137-147. 